\title{
Synthesis and Properties of Dehydroabietyl Glycidyl Ether Grafted Hydroxypropyl Chitosan
}

\author{
Wei Guo, ${ }^{\text {a,b }}$ Zhaosheng Cai, ${ }^{\mathrm{a}, *}$ Qi Xu, ${ }^{\mathrm{a}}$ Kaiyong Sun, ${ }^{\mathrm{a}}$ Xujuan Huang, ${ }^{\mathrm{a}}$ and Zhi Cao ${ }^{\mathrm{a}}$
}

\begin{abstract}
A nonionic surfactant based on chitosan and rosin, i.e. dehydroabietyl glycidyl ether grafted hydroxypropyl chitosan (DAGE-g-HPCS), was synthesized for the first time through reaction between hydroxypropyl chitosan (HPCS) and dehydroabietyl glycidyl ether (DAGE). The surface activities of DAGE-g-HPCS in aqueous solution were investigated via determination of surface tension, and its emulsifying ability was evaluated according to the stability time of emulsion composed of benzene-water with DAGE-g-HPCS as emulsifier; the foam stability was evaluated using the oscillation method. Through modulating the molecular weight of HPCS and the grafting degree (DG) of DAGE-g-HPCS, the emulsion stability and foam stability were surprisingly controllable. Compared to HPCS and specific emulsifier, the stability time of emulsion composed of benzenewater with DAGE-g-HPCS showed a remarkable performance with DG of $16.5 \%$ as the emulsifier was $7320 \mathrm{~s}$, and the foam retention of DAGE-gHPCS with $2.0 \mathrm{~g} \cdot \mathrm{L}^{-1}$ was $91.5 \%$. Simultaneously, the critical micelle concentration (CMC) of DAGE-g-HPCS decreased with increased DG, while the emulsifying ability and foam stability exhibited opposite trend. The CMC, emulsifying ability, and foam stability of DAGE-g-HPCS increased with decreased HPCS molecular weight. These results demonstrated that the DAGE-g-HPCS was an excellent surfactant that should be considered as a promising substitute for petrochemical nonionic surfactant.
\end{abstract}

Keywords: Surfactant; Chitosan; Emulsifying ability; Foam stability

Contact information: a: School of Chemistry and Chemical Engineering, Yancheng Institute of Technology, Yancheng 224051, Jiangsu, People's Republic of China; b: School of Chemistry and Chemical

Engineering, Jiangsu University, Zhenjiang 212000, Jiangsu, People's Republic of China;

*Corresponding author: jsyc_czs@163.com

\section{INTRODUCTION}

Rosin is a mixture of resin acids including different abietic and primary acids that have hydrophenanthrene structures, a certain quantity of fatty acids, and a few other neutral components (Maiti et al. 1989; Botham et al. 2008; Silvestre and Gandini 2008). Rosin is a biodegradable and biocompatible compound, and oral toxicity studies on animals have shown rosin to be practically non-toxic (Lin et al. 2018). The presence of a hydrophenanthrene ring in the structure of dehydroabietic alcohol could endow it with hydrophobicity and rigidity, and make it possible to prepare a surfactant with dehydroabietic alcohol as the chemical to form a hydrophobic group (Liang et al. 2018; Jia et al. 2019). Rosin can also be modified and grafted to obtain Gemini surfactants. First, rosin acid reacts with ethanol to obtain ethyl rosinate, which is then modified with fumaric acid. Finally, the modified ethyl rosinate is grafted with an epoxy quaternary ammonium salt to obtain a rosin-based Gemini surfactant. Maleic anhydride propyl chloride can be synthesized by the reaction of maleic acid with chlorobenzyl ester, which then reacts with 
epichlorohydrin to form 2-hydroxy-3-L-maleic anhydride propyl chloroacetate, and finally 2-hydroxy-3-L-maleic anhydride propyl chloroacetate reacts with triethylamine to obtain bis-N-(3-levoperic acid maleate adduct-2-hydroxy)propyl-triethylammonium, a rosinbased cationic surfactant (Chen et al. 2012).

Chitosan (CTS) is a type of natural macromolecule with biocompatibility, biodegradability, nontoxicity, and renewability, etc. CTS is obtained by deacetylation of chitin, which is the second most abundant biopolysaccharide in nature, next to cellulose. The chitosan-based surfactants could be synthesized by grafting of chitosan's hydrophilic groups with hydrophobic groups (Yoshioka et al. 1995; Lee et al. 2005a, 2005b; Babak and Desbrieres 2006; Chatterjee et al. 2010; Gârlea et al. 2010; Negm and Ali 2010; Chatterjee et al. 2011; Bharmoria et al. 2013; Richardson et al. 2013; Atta et al. 2014; Scheeren et al. 2016; Li et al. 2018; Senra et al. 2018; Yang et al. 2018). The N-mercapto chitosan with different alkyl chain lengths could be obtained by the reaction between chitosan and glucosamine residue in the presence of high anhydride (Lasareva et al. 2018). Chitosan could also be depolymerized into low-molecular weight chitosan, then react with an aldehyde-terminated polyethylene oxide that was formed by an oxidizing hydroxy group at the terminal of the hydrophilic polyethylene oxide to synthesis chitosan-based surfactant in the presence of sodium cyanoborohydride (Mai-Ngam 2006).

However, there has been no report yet concerned with the preparation of a surfactant based on hydroxypropyl chitosan and dehydroabietic alcohol. Herein, the authors report a novel nonionic polymeric surfactant, dehydroabietic glycidyl ether grafted hydroxypropyl chitosan (DAGE-g-HPCS) with good emulsifying properties (the stability time of emulsion composed of benzene-water with DAGE-g-HPCS as the emulsifier was $7318 \mathrm{~s}$ ) and foam stability (the foam retention of DAGE-g-HPCS with aqueous solution of $2.0 \mathrm{~g} \cdot \mathrm{L}^{-1}$ was $91.5 \%$ ), which were obtained by the grafting reaction between dehydroabietic glycidyl ether (DAGE), a derivative of dehydroabietic alcohol, and hydroxypropyl chitosan (HPCS).

\section{EXPERIMENTAL}

\section{Materials}

Chitosan was purchased from Sinopharm Chemical Reagent Co., Ltd. (Shanghai, China). Its degree of deacetylation was $88.95 \%$. Sodium hydroxide $(\mathrm{NaOH})$ and isopropanol were analytical grade and purchased from Shanghai Lingfeng Chemical Reagent Co., Ltd. (Shanghai, China). Propylene oxide was analytical grade and purchased from Shanghai Zhanyun Chemical Co., Ltd. (Shanghai, China). Acetone was analytical grade and purchased from Shanghai Shenxiang Chemical Reagent Co., Ltd. (Shanghai, China). Anhydrous ethanol was purchased from Sinopharm Chemical Reagent Co., Ltd. (Shanghai, China) and was analytically pure.

\section{Analytical Methods}

The Fourier transform infrared (FT-IR) spectra were measured on a Jasco-4100 (JASCO Co., Ltd., Tokyo, Japan) with $\mathrm{KBr}$ disks. The hydrogen-1 nuclear magnetic resonance ( ${ }^{1} \mathrm{H}$ NMR) was measured on a Bruker AVIII-500 spectroscope with TCI CryoProbe (Bruker Tech. and Serv. Co., Ltd., Beijing, China). Elemental analysis (C, H, and $\mathrm{N}$ ) were performed on a FLASH 2000 Vario micro elemental analyzer (THERMO Inc., Waltham, MA, USA). The ultraviolet-visible (UV-Vis) spectra were measured on a UV- 
5500 PC ultraviolet visible spectrophotometer (METASH Co., Ltd., Shanghai, China). The degree of substitution (DS) and grafting degree of (DG) were calculated based on the elemental analysis results.

\section{Synthesis of HPCS}

The CTS was alkalized with $30 \% \mathrm{NaOH}$ solution for 4 days. Then, $20.0 \mathrm{~g}$ alkalized chitosan, $180 \mathrm{~mL}$ isopropanol, and $141.6 \mathrm{~g}$ propylene oxide were added to a flask and stirred at $50{ }^{\circ} \mathrm{C}$ for $36 \mathrm{~h}$. The reacted material was filtered, and the solids were taken out and dissolved in $500 \mathrm{~mL}$ of distilled water. The obtained solution was placed in a dialysis bag and dialyzed in distilled water for $24 \mathrm{~h}$ before it was concentrated under reduced pressure. The concentrated mixture was precipitated with acetone, and the precipitate was collected by filtration. Finally, the obtained precipitate was freeze-dried to yield a pale yellow HPCS powder.

A total of $4 \mathrm{~g} \mathrm{HPCS}, 150 \mathrm{~mL}$ of distilled water, $250 \mathrm{~mL}$ of $2 \% \mathrm{HCl}$ solution, and an appropriate amount of $8 \%$ sodium nitrite solution were added to a flask and stirred at 60 ${ }^{\circ} \mathrm{C}$ for $0.5,1,1.5,2$, and $3 \mathrm{~h}$. Then, the $\mathrm{pH}$ of the resulting solution was adjusted to $7.5 \mathrm{with}$ aqueous ammonia, and $0.8 \mathrm{~g}$ sodium borohydride was added to the solution and stirred at $35{ }^{\circ} \mathrm{C}$ for $20 \mathrm{~min}$. The obtained mixture was then concentrated and precipitated with acetone, finally, the obtained precipitate was freeze-dried to give a different molecular weight HPCS.

\section{Synthesis of DAGE and DAGE-g-HPCS}

A total of $10 \mathrm{~g}$ dehydroabietic alcohol, $80 \mathrm{~mL}$ toluene, and metered $\mathrm{BF}_{3}$-ether were added to a flask, the metered epichlorohydrin was added dropwise, and the temperature of the material was controlled below $25^{\circ} \mathrm{C}$ during the dropping process. Then the resulting mixture was stirred at $65{ }^{\circ} \mathrm{C}$ for $4 \mathrm{~h}$, and an appropriate amount of toluene and $4.5 \mathrm{~g} \mathrm{NaOH}$ solution with mass fraction of $5 \%$ were added to the resulting mixture and stirred at $35{ }^{\circ} \mathrm{C}$ for $5 \mathrm{~h}$. After alkalization treatment, the obtained mixture was washed with water to neutral, and dehydrated with anhydrous sodium sulfate to remove toluene to obtain the light yellow DAGE oil-like liquid.

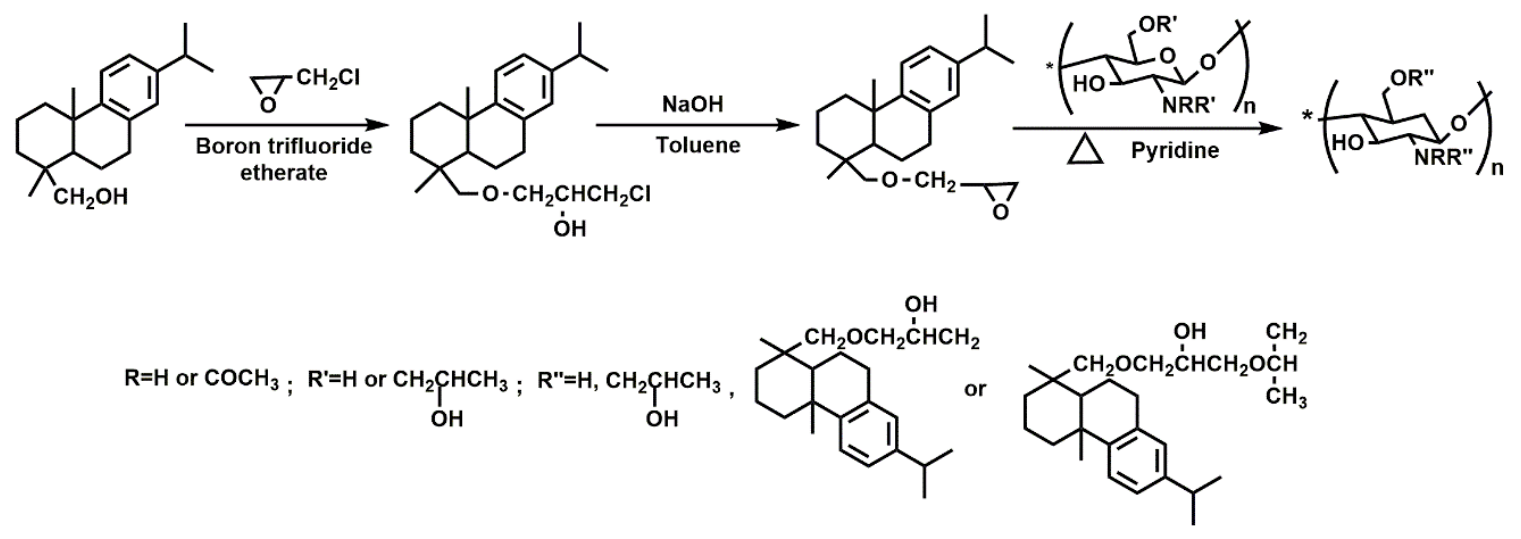

Fig. 1. The synthetic routes for the preparation of DAGE-g-HPCS

Next, 2 g HPCS, $60 \mathrm{~mL}$ pyridine, and a certain amount of DAGE were added to a flask and stirred at $50{ }^{\circ} \mathrm{C}$ for $4 \mathrm{~h}$. The obtained mixture was then concentrated under reduced pressure to remove most of the pyridine and precipitated with acetone. Then the 
resulting product was then dissolved in $300 \mathrm{~mL}$ distilled water and dialyzed for $24 \mathrm{~h}$. The dialysate was concentrated under reduced pressure and precipitated with acetone, finally, the precipitated product was extracted using a Soxhlet extractor with acetone for $8 \mathrm{~h}$. The obtained precipitate was freeze-dried to give a pale yellow DAGE-g-HPCS powder. The synthesis route of DAGE-g-HPCS is shown in Fig. 1.

\section{Elemental Analysis of HPCS and DAGE-g-HPCS}

The total amount of C and N of HPCS and DAGE-g-HPCS was measured by elemental analysis, and the $\mathrm{C} / \mathrm{N}$ ratio was calculated. Then, the DS value of HPCS and DG value of DAGE-g-HPCS was calculated by the following equation based on the $\mathrm{C} / \mathrm{N}$ ratio and DD value:

$$
\begin{aligned}
& D S=\frac{7 \times \frac{C}{N}+12 D D-48}{18} \\
& D G=\frac{7 \times \frac{C}{N}-48+12 D D-18 D S}{138}
\end{aligned}
$$

The $\mathrm{C} / \mathrm{N}$ is the ratio of total carbon to total nitrogen in the product, $D D$ is the degree of deacetylation of chitosan, $D S$ is the degree of hydroxypropyl substitution of HPCS, and $D G$ is the grafting degree of DAGE-g-HPCS.

\section{Surface Activity of DAGE-g-HPCS}

The DAGE solution of $2 \mathrm{gL}^{-1}$ was diluted to different concentrations of $1 \mathrm{~g} \mathrm{~L}^{-1}, 0.5$ $\mathrm{g} \mathrm{L}^{-1}, 0.25 \mathrm{~g} \mathrm{~L}^{-1}, 0.125 \mathrm{~g} \mathrm{~L}^{-1}, 0.062 \mathrm{~g} \mathrm{~L}^{-1}, 0.031 \mathrm{~g} \mathrm{~L}^{-1}$, and $0.016 \mathrm{~g} \mathrm{~L}^{-1}$. The surface tension $(\gamma)$ of all the DAGE-g-HPCS solutions at $24{ }^{\circ} \mathrm{C}$ was determined by the pull ring method (Chen et al. 2011), and the $\gamma$ of each concentration sample was measured three times and averaged. The $\gamma-\log C$ relationship (Origin Corporation, OriginLab, v.9.0, Northampton, MA, USA) curve was made to determine the critical micelle concentration (CMC), the surface tension value corresponding to $\mathrm{CMC}\left(\gamma_{\mathrm{CMC}}\right)$ and the minimum surface tension $\left(\gamma_{\mathrm{min}}\right)$ of DAGE-g-HPCS.

\section{Emulsifying Ability of DAGE-g-HPCS}

The $0.25 \mathrm{~g}$ DAGE-g-HPCS was dissolved in $50 \mathrm{~mL}$ distilled water and filtered through a G3 sand core funnel. Then $40 \mathrm{~mL}$ of the filtrate and $40 \mathrm{~mL}$ benzene were transferred into a 100-mL stoppered cylinder. The obtained mixture stood for 2 min after shaking up and down vigorously 20 times, and the above operation was repeated 5 times. The time used to separate $10 \mathrm{~mL}$ of water from benzene-aqueous emulsion was measured three times, and the average of the three sets of data was taken as the emulsion stabilization time.

\section{Foam Stability of DAGE-g-HPCS}

A total of $100 \mathrm{~mL}$ DAGE-g-HPCS solution with the concentration of $2.0 \mathrm{~g} \mathrm{~L}^{-1}$ was prepared, and then $50 \mathrm{~mL}$ of the prepared solution was transferred to a $100-\mathrm{mL}$ stoppered cylinder. The resulting mixture was vigorously shaken up and down 50 times, and the foam height at $0 \mathrm{~min}$ and $5 \mathrm{~min}$ were recorded. The ratio of the foam height at $5 \mathrm{~min}$ to $0 \mathrm{~min}$ was taken as the foam retention of the surfactant. The above operation was repeated three 
times to obtain three sets of data and the average value was taken as a measure of foam stability of the surfactant.

\section{RESULTS AND DISCUSSIONS}

\section{Chemical Characterization}

The results of DS of HPCS and DG of DAGE-g-HPCS are shown in Table 1.

Table 1. The DS of HPCS and DG of DAGE-g-HPCS

\begin{tabular}{|c|c|c|c|c|c|}
\hline Sample Name $^{\mathrm{a}}$ & $\begin{array}{c}\text { Content of C } \\
(\%)^{\mathrm{b}}\end{array}$ & $\begin{array}{c}\text { Content of N } \\
(\%)^{\mathrm{c}}\end{array}$ & $\mathrm{C} / \mathrm{N}$ & DS & DG \\
\hline HPCS & 41.49 & 5.69 & 7.30 & 0.76 & \\
\hline DAGE-g-HPCS1 & 42.16 & 4.71 & 8.96 & 0.76 & 0.0538 \\
\hline DAGE-g-HPCS2 & 40.77 & 4.88 & 8.36 & 0.76 & 0.0844 \\
\hline DAGE-g-HPCS3 & 43.39 & 4.61 & 9.40 & 0.76 & 0.1071 \\
\hline DAGE-g-HPCS4 & 44.61 & 4.47 & 9.97 & 0.76 & 0.1360 \\
\hline DAGE-g-HPCS5 & 45.76 & 4.36 & 10.55 & 0.76 & 0.1654 \\
\hline DAGE-g-HPCS I & 40.87 & 4.80 & 8.51 & 0.76 & 0.0618 \\
\hline DAGE-g-HPCS II & 41.98 & 4.96 & 8.46 & 0.76 & 0.0593 \\
\hline DAGE-g-HPCS III & 42.90 & 5.01 & 8.55 & 0.76 & 0.0639 \\
\hline DAGE-g-HPCS IV & 43.07 & 5.15 & 8.36 & 0.76 & 0.0542 \\
\hline DAGE-g-HPCS V & 43.73 & 5.13 & 8.52 & 0.76 & 0.0627 \\
\hline
\end{tabular}

a Samples from DAGE-g-HPCS1 to DAGE-g-HPCS5 are defined by the feed of DAGE/HPCS, the number behind HPCS represents the feed molar percentage of DAGE/HPCS. Samples from DAGE-g-HPCS I to DAGE-g-HPCS V are defined by the molecular weight of HPCS, the number behind HPCS from I to V represents the molecular weight of HPCS from high to low;

b, c Calculated from elemental analysis

The FT-IR spectra of CTS, HPCS, and DAGE-g-HPCS are shown in Fig. 2. In the FT-IR spectrum of CTS, the absorption peaks of 1639 and $1316 \mathrm{~cm}^{-1}$ represent amide I and amide III, respectively. The $3426 \mathrm{~cm}^{-1}$ represents the stretching vibration absorption peaks of $\mathrm{O}-\mathrm{H}$ and $\mathrm{N}-\mathrm{H} ; 1385 \mathrm{~cm}^{-1}$ represents the $\mathrm{C}-\mathrm{H}$ bending vibration peak of the methyl group; $1156 \mathrm{~cm}^{-1}$ reflects the absorption peak of the oxygen bridge; and $1426 \mathrm{~cm}^{-1}$ reflects the $\mathrm{C}$ $\mathrm{H}$ deformation vibration and bending vibration absorption of the methyl group and the methylene group. The 1053 and $1083 \mathrm{~cm}^{-1}$ signals reflect the C-O stretching vibration absorption peak, and the peaks at 2971 and $2924 \mathrm{~cm}^{-1}$ are C-H stretching vibration absorption peaks reflecting methyl and methylene groups (Boamah et al. 2015; Skwarczynska et al. 2019; Zhang et al. 2019).

Compared to CTS, in the FT-IR spectrum of HPCS, the peak intensities at 2971 $\mathrm{cm}^{-1}, 2924 \mathrm{~cm}^{-1}$, and $1457 \mathrm{~cm}^{-1}$ noticeably increased, and the appearance of a strong absorption peak at $1367 \mathrm{~cm}^{-1}$, ascribing to stretching vibration of $\mathrm{C}-\mathrm{H}$, indicated the presence of more methylene groups in the HPCS structure. Meanwhile, an absorption peak appeared at $1075 \mathrm{~cm}^{-1}$ that was ascribed to the stretching vibration of $\mathrm{C}-\mathrm{O}$ in the secondary hydroxyl group, and an absorption peak that appeared at $1303 \mathrm{~cm}^{-1}$ was ascribed to the stretching vibration of $\mathrm{C}-\mathrm{N}$ in the secondary amine structure. Hence, the hydroxypropyl 
groups are replaced on both hydroxyl groups and amino groups of CTS (Zhu et al. 2017; Kazemi et al. 2019).

In the FT-IR spectrum of DAGE-g-HPCS, the intensity of absorption peaks at 2927 and $2833 \mathrm{~cm}^{-1}$ ascribed to methyl and methylene groups remarkably increased, which showed that more groups were present in the DAGE-g-HPCS structure. The absorption peak that appeared at $1606 \mathrm{~cm}^{-1}$ was ascribed to the aromatic ring structure, demonstrating that the dehydroabietyl groups were successfully introduced into the HPCS structure. Meanwhile, the absorption peak at $1264 \mathrm{~cm}^{-1}$ was ascribed to the stretching vibration of C$\mathrm{O}$ in a secondary alcohol structure or an ether structure (Niu et al. 2018; Singh et al. 2018; Lu et al. 2019).
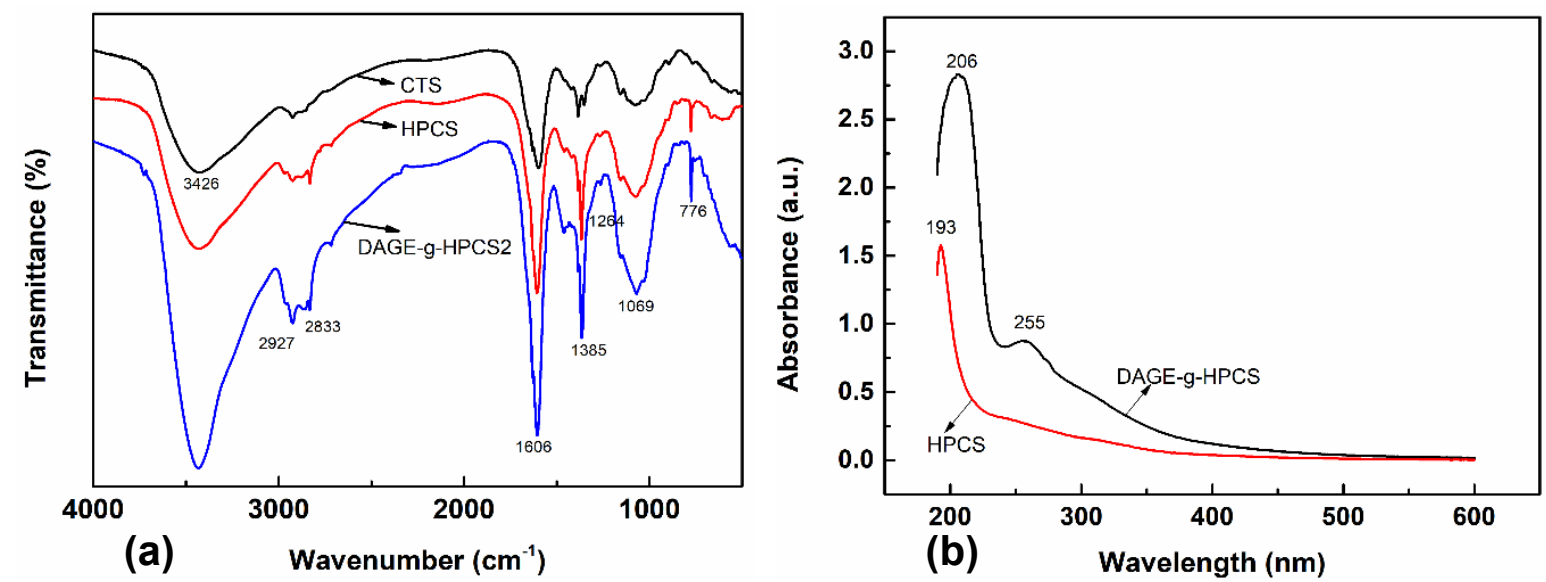

Fig. 2. The FT-IR spectra of CTS, HPCS, and DAGE-g-HPCS2 (a); UV spectrum of HPCS and DAGE-g-HPCS2 (b)

From the UV spectra of HPCS and DAGE-g-HPCS2, the HPCS solution exhibited a strong absorption peak at $193 \mathrm{~nm}$ with a narrow shape, while for the DAGE-g-HPCS2 it shifted to the right $13 \mathrm{~nm}$ compared with HPCS and appeared at $206 \mathrm{~nm}$. Additionally, a new absorption peak was also formed at $255 \mathrm{~nm}$, which indicated that DAGE had been successfully grafted onto HPCS and the graft site was mainly on the amino groups.

In the ${ }^{1} \mathrm{H}$ NMR spectra of HPCS (Fig. 3), $4.42 \mathrm{ppm}$ was assigned to $\mathrm{H}$ of $\mathrm{C}-1$ in glucosamine (Glc N) or N-acetylglucosamine (Glc NAc ) units, $3.86 \mathrm{ppm}$ was assigned to $\mathrm{H}$ of C-5 in O-substituted Glc N or Glc NAc units, 3.86 to $3.06 \mathrm{ppm}$ peaks were assigned to $\mathrm{H}$ of C-3, C-6, C-2, and methylene of O-hydroxypropyl in Glc NAc unit, signals of 3.06 to 2.63 ppm were assigned to $\mathrm{H}$ of $\mathrm{C}-6$ in the O-hydroxypropyl sugar unit, and the $\mathrm{H}$ of $\mathrm{C}$ 2, C-4 in the Glc N unit. Signals from 2.46 to $1.97 \mathrm{ppm}$ were assigned to the $\mathrm{H}$ of methylene attached to $\mathrm{N}$ in the $\mathrm{N}$-hydroxypropyl groups, the $\mathrm{H}$ of hydroxyl, and the acetyl group in Glc NAc.

In the ${ }^{1} \mathrm{H}$ NMR spectrum of DAGE-g-HPCS, besides the proton peaks of N,OHPCS, the peaks of $\mathrm{H}$ of the aromatic ring in the dehydroabietic group appeared at 8.42, 7.79, and $7.35 \mathrm{ppm}$. Meanwhile, the peaks of $\mathrm{H}$ in the groups, such as $\mathrm{CH}_{2}$ and $\mathrm{CH}_{3}$ in the dehydroabietyl group, appeared at 1.98 to $1.07 \mathrm{ppm}$, which further showed that DAGE had been successfully grafted onto N,O-HPCS (Lin et al. 2015; Lu et al. 2018; Dong et al. 2019; Xu et al. 2019). 

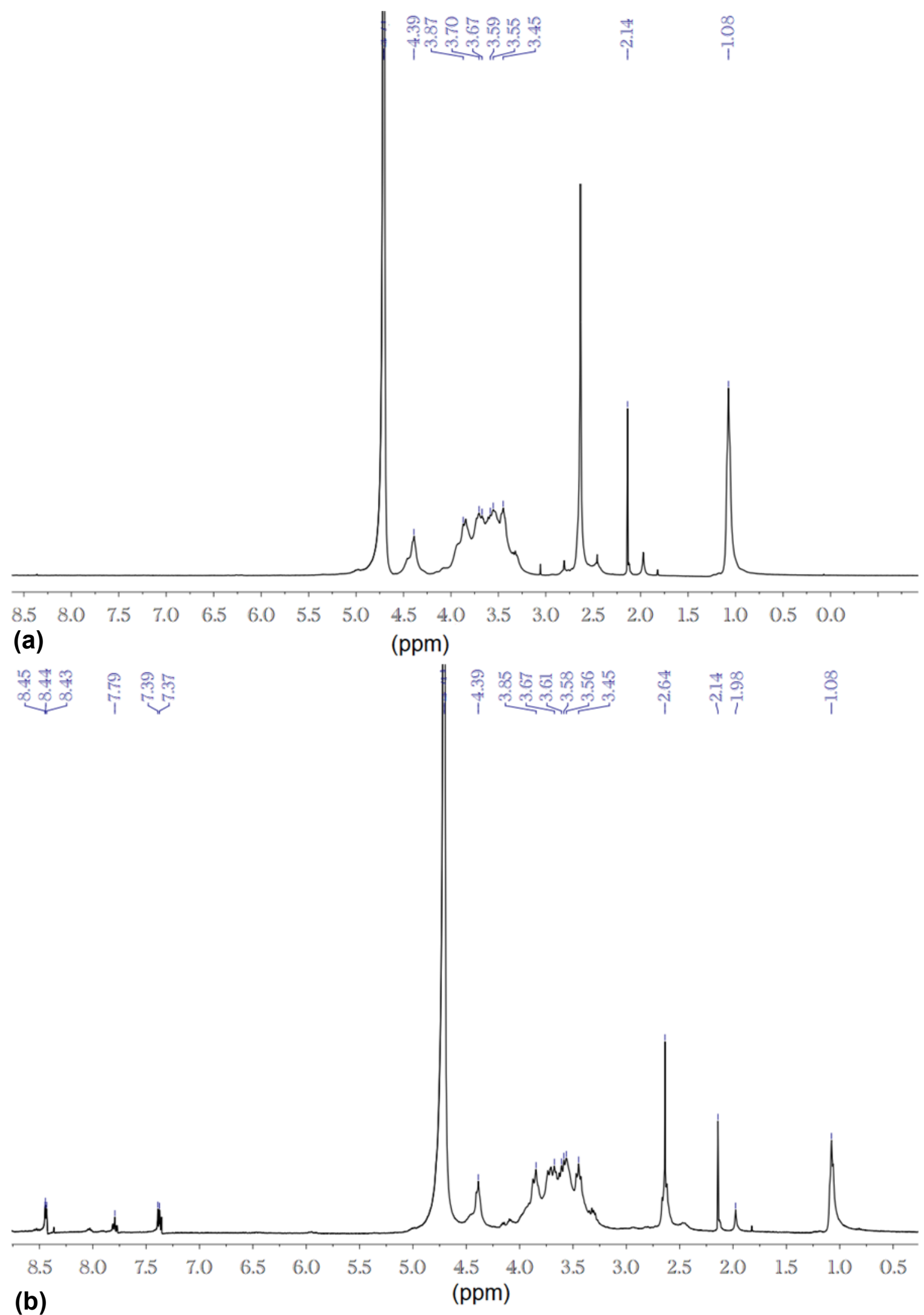

Fig. 3. The ${ }^{1} \mathrm{H}$ NMR spectrum of HPCS (a); the ${ }^{1} \mathrm{H}$ NMR spectrum of DAGE-g-HPCS2 (b) 


\section{Surface Activity}

In Fig. 4, the CMC value of DAGE-g-HPCS decreased with increased DG value, while the $\gamma_{\text {CMC }}$ value of DAGE-g-HPCS did the opposite. The $\gamma_{\text {min }}$ values of DAGE-gHPCS with different DG values were almost the same. This phenomenon was mainly related to the DG value of DAGE-g-HPCS. A larger DG value resulted in more dehydroabietyl groups that were introduced into HPCS, which enhanced the hydrophobicity of DAGE-g-HPCS. Meanwhile, enhanced hydrophobicity of DAGE-gHPCS led to the easier migration of DAGE-g-HPCS in aqueous solution, which in turn gave DAGE-g-HPCS a stronger ability to form micelles in aqueous solution even at low concentrations. The $\gamma_{\min }$ value of DAGE-g-HPCS solution was not only related to the DG value but also the rigidity of the dehydroabienyl group and the hydrophilicity of the sugar chain.

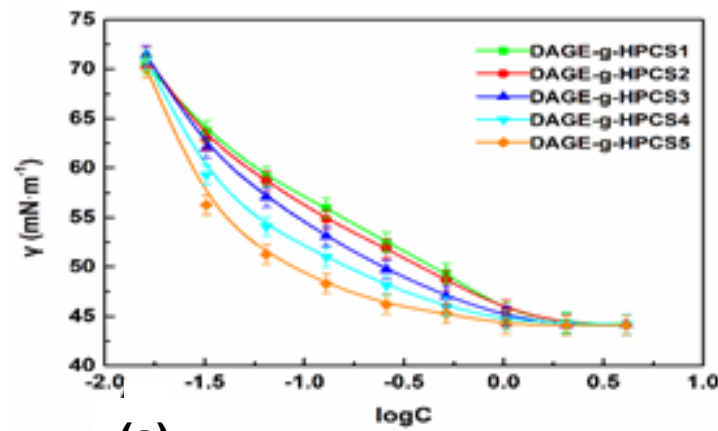

(a)

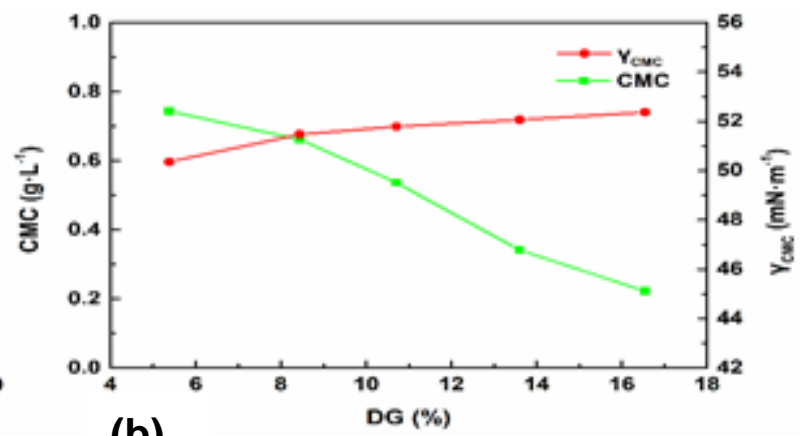

(b)

Fig. 4. $\gamma$ - $\log C$ curves of DAGE-g-HPCS with different DG (a); the relationship curves between CMC, YcMC, and Ymin of DAGE-g-HPCS and DG (b)

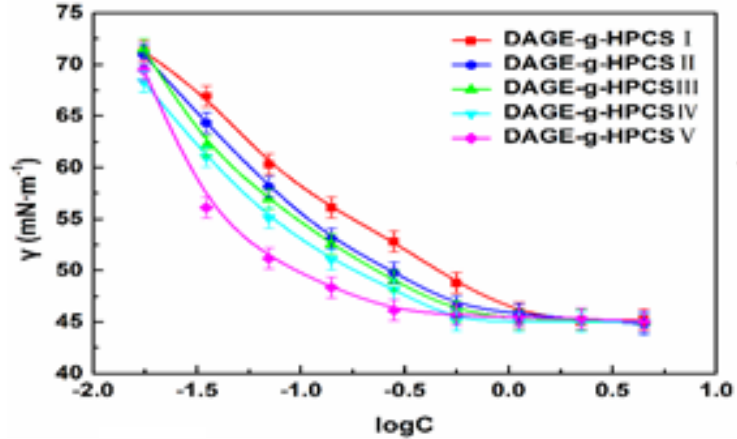

(a)

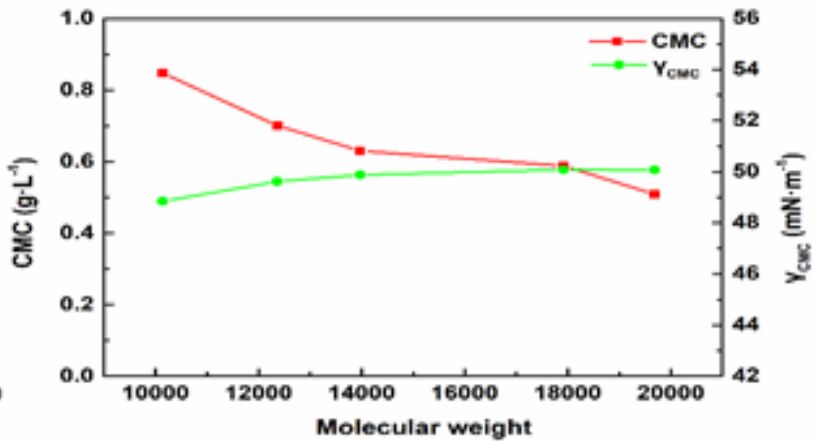

(b)

Fig. 5. $y$-logC curves of DAGE-g-HPCS with different molecular weight (a); the relationship curves between CMC, $\gamma_{\mathrm{CMC}}$, and $\gamma_{\min }$ of DAGE-g-HPCS and molecular weight of HPCS (b)

In Fig. 5, it is apparent that the CMC and $\gamma_{\mathrm{CMC}}$ values of DAGE-g-HPCS decreased with the decreasing molecular weight of HPCS, but the $\gamma_{\text {min }}$ values of DAGE-g-HPCS were almost stable in the range of $45.6 \pm 0.5$. 
The phenomenon that appeared above was mainly related to molecular weight values of DAGE-g-HPCS: in the case of similar DG, the hydrophilicity of DAGE-g-HPCS increased with the increasing molecular weight of HPCS, which mainly was due to the high-molecular weight DAGE-g-HPCS. The large molecules moved slowly by diffusion in aqueous solution, causing the hydrophobic groups to aggregate with each other to form micelles with difficulty. The decrease in molecular weight of HPCS also made the volume and the steric hindrance of HPCS decrease, leading to the increase in reactive sites of HPCS. Then, the increased reactive sites of HPCS made a more reasonable distribution of hydrophobic and hydrophilic groups in the DAGE-g-HPCS structure, which further gave DAGE-g-HPCS a stronger ability to reduce the surface tension of aqueous solution. Simultaneously, the $\gamma_{\min }$ values of DAGE-g-HPCS were stable due to the low DG (Wang et al. 2018).

\section{Emulsifying Ability}

As shown in Fig. 6, the emulsifying abilities of the whole series of DAGE-g-HPCS to benzene-water emulsion were much better than those of sucrose stearate, monoglyceride, and AEO-9. A larger DG value resulted in more dehydroabietyl groups that were grafted onto HPCS, causing enhanced surface activity of DAGE-g-HPCS and lower interfacial tension of benzene- water. Meanwhile, the strength of the benzene-water interface adsorption layer was higher because the rigidity of DAGE-g-HPCS structure was enhanced when the DAGE-g-HPCS molecule migrated to the benzene-water interface. All of the above factors were beneficial to improving the stability of the benzene-water emulsion. Meanwhile, with the decreasing molecular weight of HPCS, the emulsion stability time for benzene-water emulsion gradually increased, which was derived from the molecular weight reduction of DAGE-g-HPCS and beneficial for its better movement and arrangement in the benzene-water interface, effectively reducing the interfacial tension of benzene-water (Wang et al. 2018).

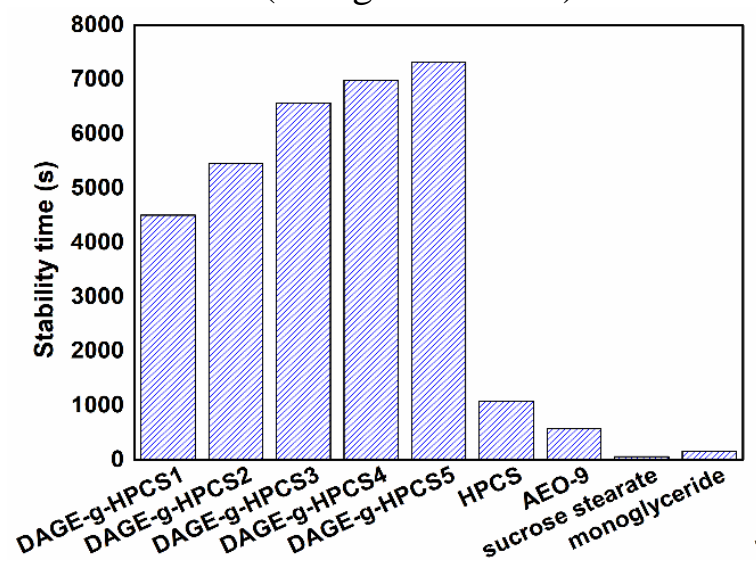

(a)

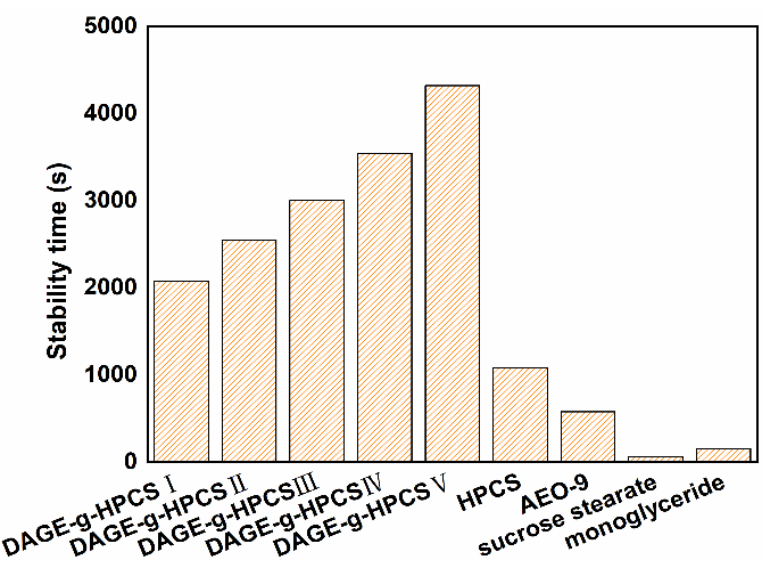

(b)

Fig. 6. Emulsifying ability of DAGE-g-HPCS with different DG and other samples to benzenewater emulsion (a); emulsifying ability of DAGE-g-HPCS with different molecular weight and other samples to benzene-water emulsion (b).

\section{Evaluation Results of Foam Stability}

The foam stability of a surfactant can be defined as the durability of the foam after the surfactant solution produces it, which is closely related to some factors such as the 
nature of the liquid film and the structure of the surfactant. In Fig. 7, the foam retention rate of DAGE-g-HPCS solution gradually increased as DG increased. This phenomenon was mainly attributable to the ternary phenanthrene ring structure of DAGE. When DAGEg-HPCS molecule was adsorbed on the surface of the bubble, the hydrophobic groups adsorbed in the liquid film increased as DG increased and enhanced the rigidity of liquid film and difficulty in draining, which further enhanced the stability of the bubble. Foam stability of DAGE-g-HPCS increased gradually as the molecular weight of HPCS decreased, which may have derived from the affinity of DAGE-g-HPCS with water that increased with the decreasing molecular weight of HPCS, resulting in lower surface tension of the solution. The surface-active molecules were adsorbed at the inner and outer surfaces of the bubble to form a bilayer film with strong stability when the foam was formed, enhancing the strength of the liquid film. Meanwhile, foam stabilities of the whole series of DAGE-g-HPCS were much better than those of sucrose stearate, monoglyceride, and fatty alcohol polyoxyethylene ether (AEO-9) (Li et al. 2003).

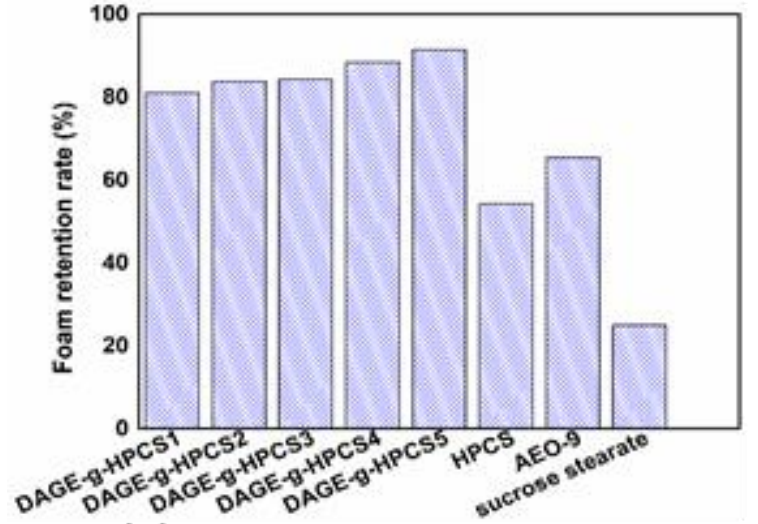

(a)

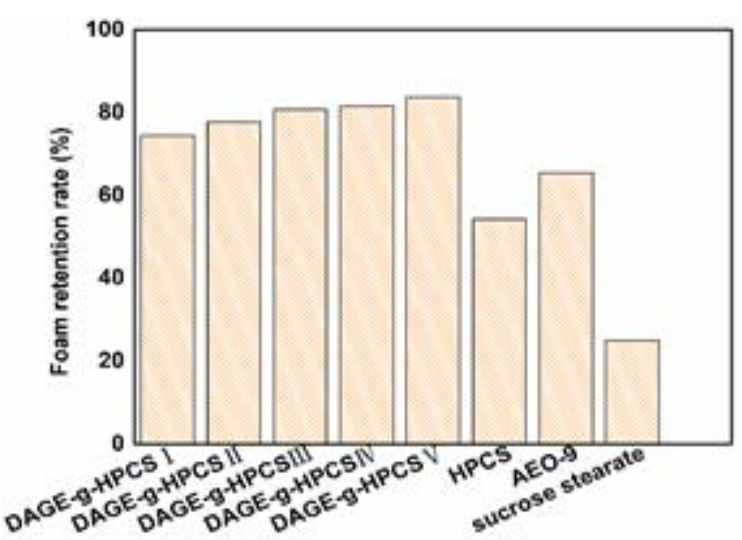

(b)

Fig. 7. Foam stability of DAGE-g-HPCS with different DG and other samples (a); the foam stability of DAGE-g-HPCS with different molecular weight and other samples (b)

\section{CONCLUSIONS}

1. In an acidic medium, hydroxypropyl chitosan (HPCS) was degraded to low-molecular weight HPCS with better water-solubility by sodium nitrite degradation, and the molecular weights of HPCS were 19700, 17900, 14000, 12400, and $10100 \mathrm{~g} / \mathrm{mole}$.

2. Through regulating the ratio of HPCS and dehydroabietyl glycidyl ether (DAGE), a nonionic surfactant DAGE-g-HPCS with different degree of grafting (DG) was obtained. The DG of DAGE-g-HPCS increased with an increasing ratio of DAGE and HPCS, and the highest DG value was $16.5 \%$. The critical micelle concentration (CMC) value of DAGE-g-HPCS aqueous solution was $0.223 \mathrm{~g} \mathrm{~L}^{-1}$, and the corresponding $\gamma_{\mathrm{CMC}}$ was $52.4 \mathrm{mN} \mathrm{m}^{-1}$. The CMC decreased as DG increased, while the emulsion stability and foam stability did the opposite.

3. Different molecular weights of DAGE-g-HPCS could be obtained by regulating the molecular weight of HPCS. The CMC, emulsion stability, and foam stability of DAGE-g-HPCS increased as its molecular weight decreased. 
4. Through comparing the emulsion stability and foam stability of DAGE-g-HPCS with that of HPCS, monoglycerides, sucrose esters, and fatty alcohol polyoxyethylene ether (AEO-9), it was found that the emulsion stability and foam stability of DAGE-g-HPCS were far better than other commonly used nonionic surfactants.

\section{ACKNOWLEDGMENTS}

The authors gratefully acknowledge the financial support of the National Natural Science Foundation of China (No. 31170543), the Jiangsu Province Key Laboratory of Biomass Energy and Materials Open Fund Project (No. JSBEM201607), the Jiangsu Province Graduate Research and Practice Innovation Program (No. SJCX18_0888), and the U.S. Department of Biomaterials Research (No. 2005-1234).

\section{REFERENCES CITED}

Atta, A. M., El-Mahdy, G. A., Al-Lohedan, H. A., and Al-Hussain, S. A. (2014). "Synthesis of environmentally friendly highly dispersed magnetite nanoparticles based on rosin cationic surfactants as thin film coatings of steel," International Journal of Molecular Sciences 15(4), 6974-6989. DOI: 10.3390/ijms15046974

Babak, V. G., and Desbrieres, J. (2006). "Dynamic surface tension and dilational viscoelasticity of adsorption layers of alkylated chitosans and surfactant-chitosan complexes," Colloid and Polymer Science 284(7), 745-754. DOI: 10.1007/s00396005-1427-x

Bharmoria, P., Singh, T., and Kumar, A. (2013). "Complexation of chitosan with surfactant like ionic liquids: Molecular interactions and preparation of chitosan nanoparticles," Journal of Colloid and Interface Science 407, 361-369. DOI: 10.1016/j.jcis.2013.06.032

Boamah, P. O., Huang, Y., Hua, M., Zhang, Q., Liu, Y., Onumah, J., Wang, W., and Song, Y. (2015). "Removal of cadmium from aqueous solution using low molecular weight chitosan derivative," Carbohydrate Polymers 122, 255-264. DOI: 10.1016/j.carbpol.2015.01.004

Botham, P. A., Lees, D., Illing, H. P. A., and Malmfors, T. (2008). "On the skin sensitisation potential of rosin and oxidised rosin," Regulatory Toxicology and Pharmacology 52(3), 257-263. DOI: 10.1016/j.yrtph.2008.05.013

Chatterjee, S., Chatterjee, T., and Woo, S. H. (2010). "A new type of chitosan hydrogel sorbent generated by anionic surfactant gelation," Bioresource Technology 101(11), 3853-3858. DOI: 10.1016/j.biortech.2009.12.089

Chatterjee, S., Chatterjee, T., and Woo, S. H. (2011). "Adsorption of Congo red from aqueous solutions using chitosan hydrogel beads formed by various anionic surfactants," Separation Science and Technology 46(6), 986-996. DOI: 10.1080/01496395.2010.538034

Chen, F., Gu, W., Zheng, Q, and Bao, M. (2011). "Discussion on the experiment "measuration of surface tension by ring-pulling method," Guangdong Chemical Industry 38(6). DOI: 208-209. 10.3969/j.issn.1007-1865.2011.06.108 
Chen, Z., Li, S., Tian, B., Liang, T., and Jin, Y. (2012). "Synthesis of a rosin gemini surfactant and its properties," Environmental Engineering Science 29(7), 606-610. DOI: 10.1089/ees.2011.0043

Dong, Y., Zhang, W., Hughes, M., Wu, M., Zhang, S., and Li, J. (2019). "Various polymeric monomers derived from renewable rosin for the modification of fastgrowing poplar wood," Composites Part B: Engineering 174, Article ID 106902. DOI: j.compositesb.2019.106902

Gârlea, A., Melnig, V., and Popa, M. I. (2010). "Nanostructured chitosan-surfactant matrices as polyphenols nanocapsules template with zero order release kinetics," Journal of Materials Science: Materials in Medicine 21(4), 1211-1223. DOI: 10.1007/s10856-009-3968-X

Jia, P., Ma, Y., Feng, G., Hu, L., and Zhou, Y. (2019). "High-value utilization of forest resources: Dehydroabietic acid as a chemical platform for producing non-toxic and environment-friendly polymer materials," Journal of Cleaner Production 227, 662674. DOI: 10.1016/j.jclepro.2019.04.220

Kazemi, M. S., Mohammadi, Z., Amini, M., Yousefi, M., Tarighi, P., Eftekhari, S., and Rafiee Tehrani, M. (2019). "Thiolated chitosan-lauric acid as a new chitosan derivative: Synthesis, characterization and cytotoxicity," International Journal of Biological Macromolecules 136, 823-830. DOI: 10.1016/j.ijbiomac.2019.06.132

Lasareva, E. V., Chernuchina, A. I., and Gabrielyan, G. A. (2018). "Preparation, surface activity and colloidal properties of the ionic complex of chitosan with hexadecyloligo-oxyethylene hemisuccinate," Carbohydrate Polymers 183, 123-130. DOI: 10.1016/j.carbpol.2017.11.089

Lee, M. Y., Hong, K. J., Kajiuchi, T., and Yang, J. W. (2005a). "Synthesis of chitosanbased polymeric surfactants and their adsorption properties for heavy metals and fatty acids," International Journal of Biological Macromolecules 36(3), 152-158. DOI: 10.1016/j.ijbiomac.2005.05.004

Lee, M. Y., Hong, K. J., Shin-Ya, Y., and Kajiuchi, T. (2005b). "Adsorption of hexavalent chromium by chitosan-based polymeric surfactants," Journal of Applied Polymer Science 96(1), 44-50. DOI: 10.1002/app.21356

Li, M., Pei, L., Cai, Z., and Fang, G. (2018). "Synthesis, characterization and properties of amphoteric polymeric surfactant CHPDMDHA-g-N,O-CMC," Chemistry and Industry of Forest Products 38(1), 115-120. DOI: 10.3969/j.issn.02532417.2018.01.017

Li, Z., Tan, H. (2003). "Foaming property and foam stability of surfactant mixed system," Oil-Gas field Surface 22(4), 13-14. DOI: 10.3969/j.issn.1006-6896.2003.04.006

Liang, H., Cheng, D., and Huang, S. (2018). "A novel rosin-based surfactant for oxidation of aromatic alcohols to ketones or aldehydes in water at room temperature," Journal of Forestry Engineering 6, 75-81. DOI: 10.13360/j.issn.20961359.2018.06.012

Lin, H. X., Yang, M. S., Tian, C., Han, C. R., Song, J., Duan, J. F., and Jiang, J. X. (2018). "Design of diversified self-assembly systems based on a natural rosin-based tertiary amine for doxorubicin delivery and excellent emulsification," Colloids and Surfaces B: Biointerfaces 165, 191-198. DOI: 10.1016/j.colsurfb.2018.01.049

Lin, R., Li, H., Long, H., Su, J., and Huang, W. (2015). "Structure and characteristics of lipase-catalyzed rosin acid starch," Food Hydrocolloids 43, 352-359. DOI:

10.1016/j.foodhyd.2014.06.008 
Lu, C., Yu, J., Wang, C., Wang, J., and Chu, F. (2018). "Fabrication of UV-absorbent cellulose-rosin based thermoplastic elastomer via "graft from" ATRP," Carbohydrate Polymers 188, 128-135. DOI: 10.1016/j.carbpol.2018.01.062

Lu, H. T., Lu, T. W., Chen, C. H., and Mi, F. L. (2019). "Development of genipincrosslinked and fucoidan-adsorbed nano-hydroxyapatite/hydroxypropyl chitosan composite scaffolds for bone tissue engineering," International Journal of Biological Macromolecules 128, 973-984. DOI: 10.1016/j.ijbiomac.2019.02.010

Mai-Ngam, K. (2006). "Comblike poly(ethylene oxide)/hydrophobic C branched chitosan surfactant polymers as anti-infection surface modifying agents," Colloids and Surfaces B: Biointerfaces 49(2), 117-125. DOI: 10.1016/j.colsurfb.2006.03.007

Maiti, S., Ray, S. S., and Kundu, A. K. (1989). "Rosin: A renewable resource for polymers and polymer chemicals," Progress in Polymer Science 14(3), 297-338. DOI: $10.1016 / 0079-6700(89) 90005-1$

Negm, N. A., and Ali, H. E. (2010). "Modification of heavy metal uptake efficiency by modified chitosan/anionic surfactant systems," Engineering in Life Sciences 10(3), 218-224. DOI: 10.1002/elsc.200900110

Niu, X., Liu, Y., Song, Y., Han, J., and Pan, H. (2018). "Rosin modified cellulose nanofiber as a reinforcing and co-antimicrobial agents in polylactic acid/chitosan composite film for food packaging," Carbohydrate Polymers 183, 102-109. DOI: 10.1016/j.carbpol.2017.11.079

Richardson, K. E., Xue, Z., Huang, Y., Seo, Y., and Lapitsky, Y. (2013). "Physicochemical and antibacterial properties of surfactant mixtures with quaternized chitosan microgels," Carbohydrate Polymers 93(2), 709-717. DOI: 10.1016/j.carbpol.2012.12.054

Scheeren, L. E., Nogueira, D. R., Macedo, L. B., Vinardell, M. P., Mitjans, M., Infante, M. R., and Rolim, C. M. B. (2016). "PEGylated and poloxamer-modified chitosan nanoparticles incorporating a lysine-based surfactant for $\mathrm{pH}$-triggered doxorubicin release," Colloids and Surfaces B: Biointerfaces 138, 117-127. DOI: 10.1016/j.colsurfb.2015.11.049

Senra, T. D. A., Campana-Filho, S. P., and Desbrières, J. (2018). "Surfactantpolysaccharide complexes based on quaternized chitosan. Characterization and application to emulsion stability," European Polymer Journal 104, 128-135. DOI: 10.1016/j.eurpolymj.2018.05.002

Silvestre, A. J. D., and Gandini, A. (2008). "Rosin: Major sources, properties and applications," in: Monomers, Polymers and Composites from Renewable Resources, M. N. Belgacem, and A. Gandini (eds.), Elsevier, Amsterdam, Netherlands, pp. 6788.

Singh, V., Joshi, S., and Malviya, T. (2018). "Carboxymethyl cellulose-rosin gum hybrid nanoparticles: An efficient drug carrier," International Journal of Biological Macromolecules 112, 390-398. DOI: 10.1016/j.ijbiomac.2018.01.184

Skwarczynska, A. L., Binias, D., Maniukiewicz, W., Modrzejewska, Z., and Douglas, T. E. L. (2019). "The mineralization effect on chitosan hydrogel structure containing collagen and alkaline phosphatase," Journal of Molecular Structure 1187, 86-97. DOI: 10.1016/j.molstruc.2019.03.034

Wang, T., Zhang T., Cai Z., Sang S., He J., and Wang Y. (2018). "Synthesis and performance of (2-hydroxyl-3-dehydrofiroxy) propyl hydroxyethyl chitosan," China Surfactant Detergent \& Cosmetics 48(4), 196-200. DOI:

10.13218/j.cnki.csdc.2018.04.003 
Xu, Z., Lou, W., Zhao, G., Zhang, M., Hao, J., and Wang, X. (2019). "Pentaerythritol rosin ester as an environmentally friendly multifunctional additive in vegetable oilbased lubricant," Tribology International 135, 213-218. DOI: 10.1016/j.triboint.2019.02.038

Yang, X., Zhang, B., Li, P., Guo, C., Li, J., and Li, Q. (2018). "Synthesis of a high surface active polymeric surfactant based on chitosan and characteristics of complexation with cetyltrimetylammonium bromide," Journal of Dispersion Science and Technology 39(10), 1485-1491. DOI: 10.1080/01932691.2017.1417135

Yoshioka, H., Nonaka, K., Fukuda, K., and Kazama, S. (1995). "Chitosan-derived polymer-surfactants and their micellar properties," Bioscience, Biotechnology, and Biochemistry 59(10), 1901-1904. DOI: 10.1271/bbb.59.1901

Zhang, J., Tan, W., Wei, L., Chen, Y., Mi, Y., Sun, X., Li, Q., Dong, F., and Guo, Z. (2019). "Synthesis of urea-functionalized chitosan derivatives for potential antifungal and antioxidant applications," Carbohydrate Polymers 215, 108-118. DOI: 10.1016/j.carbpol.2019.03.067

Zhu, C., Zou, S., Rao, Z., Min, L., Liu, M., Liu, L., and Fan, L. (2017). "Preparation and characterization of hydroxypropyl chitosan modified with nisin," International Journal of Biological Macromolecules 105, 1017-1024. DOI: 10.1016/j.ijbiomac.2017.07.136

Article submitted: November 29, 2019; Peer review completed: March 8, 2020; Revised version received and accepted: April 8, 2020; Published: April 10, 2020.

DOI: $10.15376 /$ biores. 15.2.4110-4123 